\title{
An Improved Bandwidth-Use Method on IEEE 802.11e Standard over Wireless LAN
}

\author{
Fang-Yie Leu, Yu-Hsin Chen, and Ching-Chien Kuan \\ Department of Computer Science and Information Engineering, \\ Tunghai University, Taichung, Taiwan \\ \{leufy,g942825\} athu.edu.tw
}

\begin{abstract}
Currently, people can collect information and share resources on the Internet through WiFi. The Voice over Internet Protocol (VoIP) supported by wireless technology has made Internet access more versatile and flexible than before. However, the more sessions established for communication, the longer the transmission delay and the higher the packet dropping rate. In this paper, an improved bandwidth utilization approach developed on the IEEE 802.11e standard is proposed. Experimental results show that this approach significantly improves a system's throughput as compared with $802.11 \mathrm{~b}$ and other schemes.
\end{abstract}

Keywords: VoIP, IEEE 802.11e, LCM, Bandwidth utilization.

\section{Introduction}

The rapid progress of scientific technology, e.g., merging different media, video, voice, images, and music, and the corresponding expansion of services, e.g., web and e-mail, will soon result in a fully digitalized world. Therefore, how to utilize the Internet technology to carry out high quality multimedia communication, especially with the human voice and video, has become one of the main challenges for research.

Due to its mobile convenience, wireless communication has become one of the focuses of researchers' attention. Several standards have been proposed, e.g., 802.11, 802.15 and 802.16. According to a report by eTForecasts [1][2], the number of people who access the worldwide network will approach 1,782 million by 2010 , and the number of wireless network users is expected to rise to 779 million by 2010 . One of the most compelling applications is VoIP. ON-World News [3] predicted that there will be more than 100 million VoIP users by 2011.

Traditional voice communication is provided by the Public Switched Telephone Network (PSTN), which deploys circuit switching to convey sound. PSTN is inefficient and expensive, whereas VoIP is efficient and inexpensive. Chen et al. [4] mentioned that today VoIP can provide all of the telephone services that traditional phone service provides. In fact, Session Initiation Protocol (SIP) [5] has been widely used in VoIP services to support call setup, call forwarding, voice mail and conference and multimedia calls. Researchers predict that SIP will be used in future all-IP mobile networks to deliver various services [6]. Wolff [7] noted that Skype membership reached 100 million at 1:12 p.m. on April 27, 2006. No other network software has reached this figure in the short span of two and a half years. The key 
reasons are that Skype membership simply requires a MIC and free access to the Internet. Courtney [8] reported that Skype was running at 30 billion Skype-to-Skype minutes a year, and predicted that the number would continue to rise. He speculated that one-tenth of a percent of those minutes go through Skype Prime. The minimum charge is $\$ 0.50$ per minute.

Generally speaking, IEEE 802.11 is only designed for best effort service [9-10]. The lack of a built-in mechanism to support real-time services makes it very difficult to guarantee QoS for throughput-sensitive and delay-sensitive multimedia applications. Hence, modification of existing 802.11 standards is necessary. Although the 802.11e has supported QoS for WLAN applications [11-12], choosing the right set of MAC parameters and the QoS mechanism to provide predictable service quality remains a challenge for researchers.

In this paper, an improved bandwidth utilization approach based on a non-packetdropping scheme developed on the IEEE 802.11e standard is proposed. It not only provides qualified transmission, but also increases communication channel utilization.

The rest of this paper is organized as follows. Section 2 introduces the related background. Section 3 describes the proposed scheme. Experimental results are described in section 4 . Section 5 concludes this paper.

\section{Background and Related Work}

\subsection{IEEE 802.11 MAC Protocol}

IEEE 802.11 MAC provides two main access methods, Distributed Coordination Function (DCF) and PCF. Coordination Function is a mechanism that coordinates when a station can start transmitting data. DCF is a basic access method, which primarily deploys Carrier-Sense Multiple Access/Collision Avoidance (CSMA/CA) to enable a station to send and receive non-synchronous data. CSMA/CA can be used in Ad Hoc and WLAN infrastructures as well. PCF, a contention-free method, enables stations to send and receive time-bounded data; hence, no packet collisions occur. However, PCF can only be used in certain basic WLAN frameworks, e.g., WLANs containing AP.

\subsection{VoIP}

VoIP Internet phone is a type of voice transmission service that first digitizes a voice signal, and then encapsulates the result into data packets for transmission over an IP network. This technique can compensate for signal distortion, echo and data loss for packet transmission so as to reestablish the original voice data and improve communication quality. Thus, utilizing the Internet not only enables real-time voice transmission services, but also achieves global connectivity, providing users with an alternative to traditional PSTN for long-distance phone calls.

\subsection{IEEE 802.11e}

IEEE 802.11e [13-16] employs Hybrid Coordination Function (HCF) as its medium access protocol. HCF uses contention-based and controlled-channel accesses as its channel allocation strategies. The former is an enhanced DCF and the latter an enhanced PCF. 
The Point Coordinator (PC) of traditional PCF is only allowed to transmit data in contention-free periods, whereas the Hybrid Coordinator ( $\mathrm{HC}$, equivalent to $\mathrm{PC}$ ) in HCF can transmit data or instruct stations to transmit data in both contention and contention-free periods. To minimize transmission delay and jitter and maximize medium transmission efficiency, IEEE 802.11e provides "packet bursting", which means that after being allowed access to a transmission medium, a station can transmit more than one frame within a certain time slot without requesting access to the transmission medium again.

\subsection{Related Work}

Using the contention approach to allocate channel access right is inadequate for transmitting real-time packets. Thus, PCF employs PC to allocate channel access right to stations. Even based on CSMA/CA, the MAC layer of IEEE 802.11b WLAN can still provide a real-time environment, but would result in less-than-optimal performance. Several solutions have been proposed [17-23]. Some give voice packets a higher priority over data packets to shorten VoIP packets' waiting time. Others suggest transferring voice packets under the DCF contention mode with special mechanisms to meet the real-time requirement. But most compromise their service quality through packet loss and delay.

PCF usually has lower channel efficiency owing to too many failed polls, especially when most stations do not attempt to transfer packets. In other words, neither PCF nor DCF is suitable for voice transmission.

\section{The Proposed Scheme}

To improve the quality of such transmission, we propose the following scheme: build a token buffer within AP, dynamically, establish a transmission polling list based on the parameters, and then follow the polling list to multi-poll stations.

\subsection{Parameters}

For each real-time station $S$, we use two parameters, $r_{c}$ and $\delta$, to represent its transmission characteristic. $r_{c}$ is the packet transfer rate, and $\delta$ the maximum amount of jitter (i.e., packet delay variation) allowed for a specific packet. Transmitting voice data either too fast or too slow should be avoided. In other words, each packet of $\mathrm{S}$ should inherit $r_{c}$ and $\delta$ from its voice source, i.e., $S$.

\subsection{Theoretical Discussion}

Assume there are $\mathrm{n}$ voice sources and their characteristic parameters are $\left(r_{c i}, \delta_{i}\right)$, $i=1,2, \cdots, n$. The maximum waiting time of a token $\mathrm{T}$, from the time point $\mathrm{T}$ 's corresponding packet $\mathrm{P}$ arrives at the transfer buffer to the time point $\mathrm{P}$ is delivered, is $\delta_{i}^{*}$.

According to theorem 1, each packet can be delivered within $\delta_{i}$. 


\section{Theorem 1[9]}

Let $\delta_{1}^{*}=2 \cdot \mathrm{SIFS}+\mathrm{CFPoll}+\mathrm{T}_{\mathrm{p}}+\mathrm{ACK}$ (See Fig. 1), and $\delta_{i}^{*}=\left(2 \cdot \mathrm{SIFS}+\mathrm{CFPoll}+\mathrm{T}_{\mathrm{p}}+\mathrm{ACK}\right)+\sum_{\mathrm{k}=1}^{\mathrm{i}-1}\left[\frac{\mathrm{r}_{\mathrm{ck}}}{\mathrm{r}_{\mathrm{ci}}}\right] \cdot\left(2 \cdot \mathrm{SIFS}+\mathrm{CFPoll}+\mathrm{T}_{\mathrm{p}}+\mathrm{ACK}\right)$, $\mathrm{i}=2, \cdots, \mathrm{n}$

where $\mathrm{T}_{\mathrm{p}}$ is the time required to transmit and receive a packet. If $\delta_{i}^{*}<\frac{1}{r_{c i}}$ and $\delta_{i}^{*} \leq \delta_{i}, \mathrm{i}=2, \cdots, \mathrm{n}$, then all voice packets of each session can be transmitted within their jitter constraints.

\section{Theorem 2 [9]}

Assume $\mathrm{n}$ voice sources are scheduled in the given priority order. The average waiting time is minimized for voice packets if $r_{c i} \leq r_{c j}$ for all $\mathrm{i}<\mathrm{j}$.

\subsection{Improvement in Multi-polling}

Improvement in multi-polling is as follows:

(1) Our design is based on a hypothetically perfect environment. AP creates a polling list in its buffer to arrange the order and relative time of packet transmission, according to the parameters $\left(r_{c i}, \delta_{i}\right)$ of the packets that have arrived at AP's transfer buffer. It then broadcasts the polling list to all stations of the underlying Basic Service Set (BSS).

\section{Theorem 3}

Given n voice sources with $r_{c i}$ and $\delta_{i}, i=1,2,3 \ldots \mathrm{n}$, there exists a cycle LCT $=\mathrm{LCM}$ (The Least Common Multiple) $\left[\frac{1}{r_{c 1}}, \frac{1}{r_{c 2}}, \ldots \ldots \ldots \ldots . . ., \frac{1}{r_{c n}}\right]$ within which the amount of packets transmitted is $\sum_{i=1}^{n}\left(r_{c i} \bullet L C T\right)$. If two or more packets of different sessions arrive at the same time point, based on theorem 2 a session with lower jitter has lower priority. This ensures a minimum total waiting time.

(2) In a BSS, stations normally follow their current polling list to transmit voice packets. AP and stations monitor whether the sequence is correct or not. If any discrepancy or collision occurs, e.g., a session is newly established or disconnected, or a station crashes or follows an out-of-date polling list, AP updates its polling list if needed and again broadcasts it to all stations which will then follow the new list. AP does not need to poll stations one by one, thus 
saving a significant amount of polling time. AP resets the TPT field at any time token buckets are empty, and judges whether it has sufficient time to switch to contention mode to transmit ordinary data. If so, AP switches to DCF mode.

\subsection{Bandwidth Utilization}

In an LCM, several tokens can be generated at the same time. Lower-priority packets are then postponed without seriously affecting their sound quality. Attempting to send voice data $D$ to another station, a station first partitions $D$ into several packets when necessary and sends them with IEEE802.11e protocol. An AP at any given moment can deploy only one channel to transmit packets. However, due to transmission overhead and times when no packets are transmitted, the channel bandwidth is very often not completely used. The former can not be avoided except by using a modified $802.11 \mathrm{e}$ protocol, which is currently unavailable in the commercial market. However, if we can negotiate with some stations which, based on theorem 1, are not allowed to send packets at first, to reduce their $r_{c i s}$ and extend their jitters, then many empty slots will be efficiently used and channel bandwidth utilization will be significantly improved. In the following subsection, two ways to increase bandwidth utilization are discussed.

\subsubsection{The Ways to Improve Channel Utilization}

Let $K=\left(k_{1}, k_{2}, k_{3}, \ldots, k_{m}\right)$ be empty slots in an LCM. Assume there is an arithmetic progression A rip $=\left\{k^{\prime}{ }_{1}, k^{\prime}{ }_{2}, \ldots, k^{\prime}{ }_{p}\right\}$ with a parameter $c$, where $c=k^{\prime}{ }_{j+1}-k^{\prime}{ }_{j}>0 \quad$ for $\quad$ all $\quad \mathrm{j}, \quad 1 \leq j \leq p-1 \quad$ and $k^{\prime}{ }_{1}=\left(k^{\prime}{ }_{p}+c\right) \% \quad p \quad$, i.e., a circular arithmetic progression.

Approach 1 is applicable to the situation where elements of Arip are all unallocated, i.e., for all $k^{\prime}{ }_{i}, k^{\prime}{ }_{i} \in K, 1 \leq i \leq p$, and a station, e.g., $S T A_{g}$, which is filtered out by theorem 1, agrees to reduce its $r_{c}$ to $r^{\prime}{ }_{c}\left(=1 /\left(c *\right.\right.$ time $\left.\left.e_{-} o f_{-} a_{-} s l o t\right)\right)$, and changes its $\delta_{c}$ to $\delta^{\prime}{ }_{c}\left(=1 / r^{\prime}{ }_{c}\right)$. We then allocate Arip to $S T A_{g}$, which will send data with $r^{\prime}{ }_{c}$ and $\delta^{\prime}{ }_{c}$. As several such Arips exist simultaneously, e.g., Arip 1 with $c_{1}=3$ and Arip 2 with $c_{2}=6$, the one with the smallest $c$ is chosen since much more data can then be sent. However, when there are several such stations, the one with the greatest $r_{c}$ will be negotiated with first.

Approach 2 is applicable to the situation where some elements (slots) of Arip have been allocated, e.g., $K O=\left\{o_{1}, o_{2}, \ldots, o_{w}\right\}$ where $o_{i} \notin K, o_{i} \in A$ rip , $i=1,2, \ldots, w$. However, for each $o_{i}$, there is at least one empty slot $k^{\prime}{ }_{b}$, located between $O i\left(=k^{\prime}{ }_{v}\right)$ and $k^{\prime}{ }_{v+1}, 1 \leq v \leq p-1, k^{\prime}{ }_{b} \in K$. STA $A_{g}$ can then transmit a packet for $S T A_{g}$ at $k^{\prime}{ }_{b}$ instead of at $k^{\prime}{ }_{v}$ without violating $S T A_{g}{ }^{\prime} s$ new jitter. 


\section{Simulations and Experiments}

In the following, we evaluate the performance of the proposed scheme.

\subsection{Simulation Environment}

The basic assumptions of our simulation environment are as follows. Two types of traffic are considered.

(1) Pure data. The arrival of data frames from a station's higher-layer to the MAC sub-layer is Poisson. Frame length is assumed to be exponentially distributed with a mean length of 1024 octets.

(2) Voice traffic. We use the mio8380's [24] built-in audio codec based on the GSM610 format to generate voice traffic patterns. For each session, frames are sent every $10 \mathrm{~ms}$. Sessions are generated as exponentially distributed. Frames of voice traffic that are not successfully transmitted within their maximum jitter constraint are assumed to be lost.

\subsection{Simulation Results}

The simulation was performed based on the number of sessions that could successfully complete their communication. We generated a fixed number of session requests with the same $r_{c}$ and jitter for each of the following groups: LCM multipolling [9], LCM single-polling [9], 802.11b [17] [20-23], 802.11e [15-16][23], and scheduled the requests of a group in a sequence of time slots.

Fig. 1 shows that channel utilization of GCD (of course, LCM-based) was not as high as that of the IEEE 802.11 b since GCD filtered out requests with theorem 1, but 802.11 b did not. The GCD outperformed the LCMs since GCD dynamically adjusted sessions' jitters to make them acceptable to theorem 1 . The bandwidth utilization was then improved.

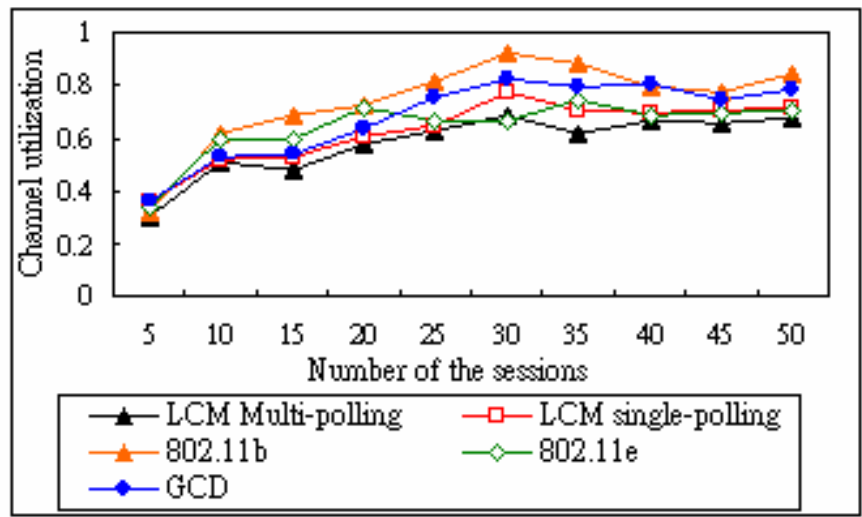

Fig. 1. Channel utilization for different sessions 


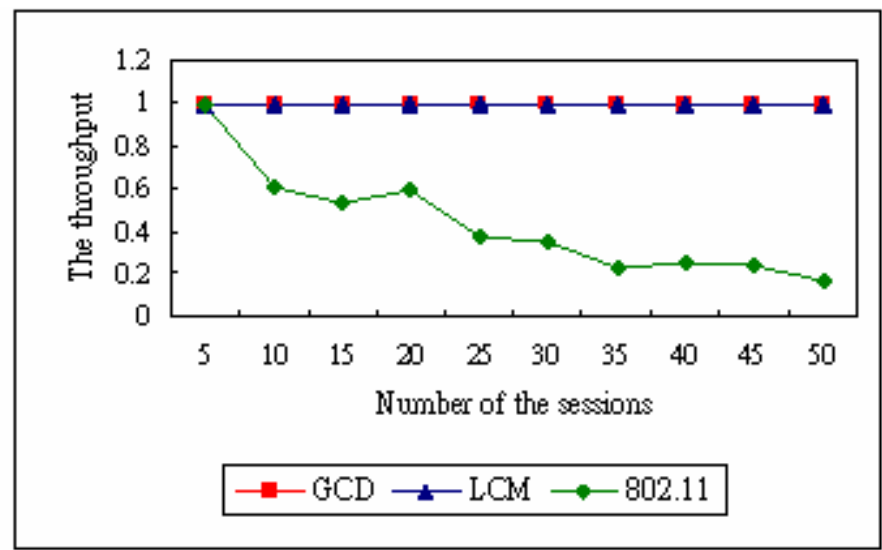

Fig. 2. Throughput for different sessions

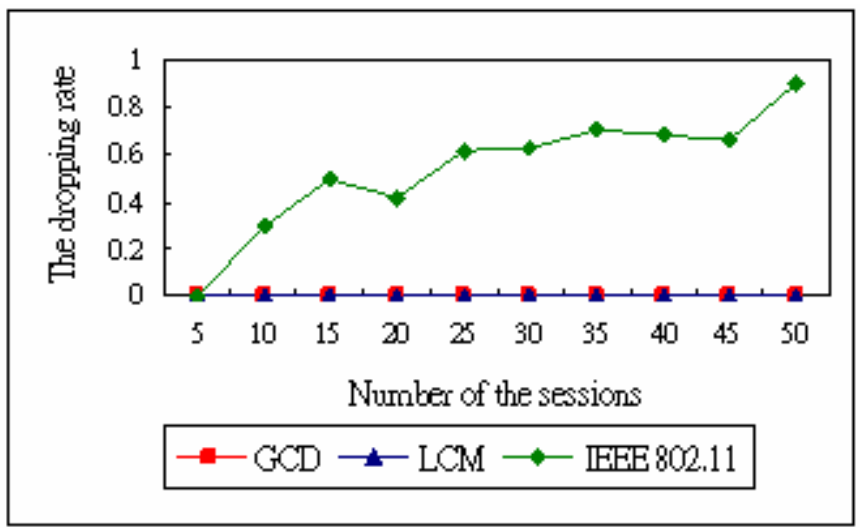

Fig. 3. Dropping rate for different sessions

We defined throughput as the ratio of successfully transmitted packets over total packets. In the IEEE 802.11 standard, all collided packets were dropped. Our mechanism will block all requests whose jitters can not be satisfied, hence dropping no packets in any sessions. The only side effect is that those stations will continuously request AP until their requests are accepted. Fig. 2 shows the simulation results.

Fig. 3 illustrates that the dropping rates of the GCD and LCM were significantly lower than that of the IEEE 802.11 standard. As stated above, both in single-polling and multi-polling, their values were zero.

Fig. 4 shows that GCD's channel utilization was not better than that of IEEE 802.11b. But when the $r_{c}$ of the requests rejected by theorem 1 was reduced, GCD was better than IEEE802.11b. 


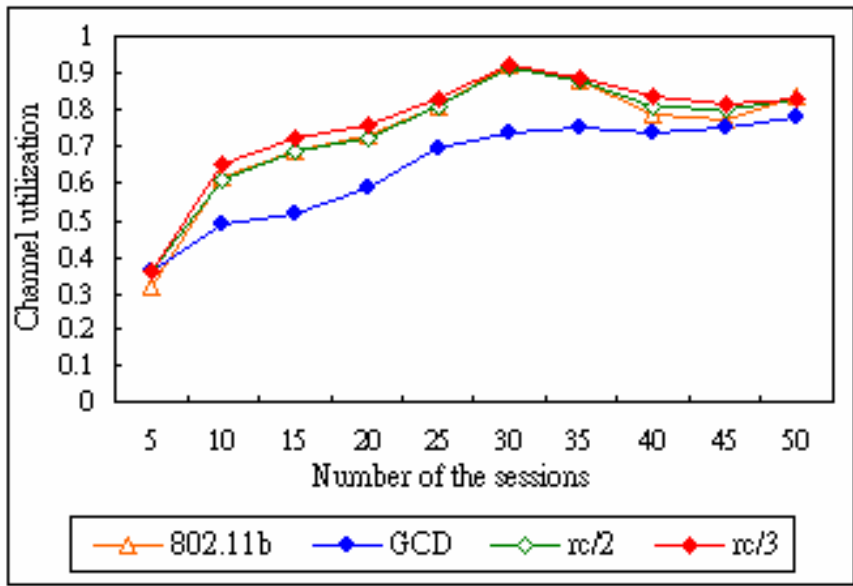

Fig. 4. Channel utilization when some sessions reduce their packet transmission rates $r_{c}$

\section{Conclusion}

Based on previous simulations, our mechanism has significantly improved packet dropping rate by checking to see whether a collision will occur. It also results in repeated requests and connection delays. However, a connection once established is packet-dropped free ensuring less delay resulting from collision. In addition, LCM does not promise maximum bandwidth utilization, but is able to predict the transmission order of packets by using a polling list to achieve Multi-Polling and meet the IEEE 802.11e standard.

Our scheme is able to control current network bandwidth by deploying a token buffer. The flexibility of controlling sessions ensures that they will be collision free. The actual bandwidth is not necessarily full, and therefore the 'packet transfer rate' and 'tolerated jitter' values become very important. Simultaneous small jitters of multiple sessions will cause a jam, thus forcing a token buffer to fill up much earlier. However, the bandwidth is not fully occupied, theoretically allowing more tolerated large-size jitters. Simultaneous large session jitters will also cause a jam, but as with small session jitters, our mechanism can increase the bandwidth by accepting more tolerated small-size jitters. In summary, the capacity of pairing the 'packet transfer rate' and 'tolerated jitter' values is a tradeoff but one of critical importance.

\section{References}

1. Worldwide Internet Users Top 1 Billion in 2005-USA Reaches Nearly 200M Internet Users, (January 3, 2006) http://www.etforecasts.com/pr/pr106.htm

2. Internet Users by Country, eTForecasts's Products. http://www.etforecasts.com/products/ES_intusersv2.htm 
3. One Hundred Million Mobile VOIP Users in 2011 (May 23, 2006) http://www.onworld.com/html/newscmvoip.htm

4. Chen, J.J., Cheng, Y.L., Tseng, Y.C., Wu, Q.: A Push-Based VoIP Service for an InternetEnabled Mobile Ad Hoc Network. In: The IEEE Asia Pacific Wireless Communications Symposium (2006), http://www.csie.nctu.edu.tw/ỹctseng/pub.html

5. Rosenberg, J., et al.: SIP:Session Initiation Protocol. IETF RFC 3261(June 2002)

6. Lin, Y.B., Huang, Y.R., Pang, A.C., Chlamtac, I.: All IP Approach for Third Generation Mobile Networks. IEEE Networks 16(5), 8-19 (2002)

7. Wolff, P.: Skype Achieves 100 Million Users (April 28, 2006), http://www.skypejournal.com/blog/archives/2006/04/ 100000000_or_so_skype_accounts_more_skyp_1.php

8. Courtney, J.: The Skype Prime Rates (March 07, 2007), http://www.skypejournal.com/blog/every_post/skype/

9. Leu, F.Y., Kuan, C.C., Deng, D.J., Chang, W.K.: Quality of Service for Voice over Wireless LAN by Deploying Multipolling in IEEE802.11e Standard. In: Proc. of International Symposium on Multimedia Over Wireless, June 2005, pp. 170-175 (2005)

10. Gu, D., Zhang, J.: QoS Enhancement in IEEE 802.11 Wireless Local Area Networks. IEEE Communication Magazine 41(6), 120-124 (2003)

11. Wang, Y., Bensaou, B.: Priority-Based Multiple Access for Service Differentiation in Wireless Ad-Hoc Networks. In: Proc. of Mobile and Wireless Communication Networks, pp. 14-31 (2000)

12. Chuang, P.H., Wu, H.K., Liao, M.K.: Dynamic QoS Allocation for Multimedia Ad Hoc Wireless Networks. In: Proc. of Computer Communications and Networks, pp. 480-485 (October 1999)

13. Ni, Q.: Performance Analysis and Enhancements for IEEE 802.11e Wireless Networks. IEEE Networks 19(4), 21-27 (2005)

14. Hwang, G.H., Cho, D.H.: New Access Scheme for VoIP Packets in IEEE 802.11e Wireless LANs. IEEE Communications Letters 9(7), 667-669 (2005)

15. Naoum-Sawaya, J., Ghaddar, B., Khawam, S., Safa, H., Artail, H., Dawy, Z.: Adaptive Approach for QoS Support in IEEE 802.11e Wireless LANs. In: Proc. of the IEEE International Conference on Wireless And Mobile Computing, Networking and Communications, vol. 2, pp. 167-173 (2005)

16. Lee, J.F., Liao, W., Chen, M.C.: A Per-Class QoS Service Model in IEEE 802.11e WLANs. Quality of Service in Heterogeneous Wired/Wireless Networks (QShine) (August 2005)

17. Sobrinho, J.L., Krishnakumar, A.S.: Quality-of-Service in Ad Hoc Carrier Sense Multiple Access Wireless Networks. IEEE Journal on Selected Areas of Communications 17(8), 1353-1368 (1999)

18. Xiao, Y.: Enhanced DCF of IEEE 802.11e to Support QoS. Proc. of Wireless Communication and Networking, pp. 1291-1296 (March 2003)

19. Eshghi, F., Elhakeem, A.K.: Performance Analysis of Ad Hoc Wireless LANs for RealTime Traffic. IEEE Journal on Selected Areas of Communications 21(2), 204-215 (2003)

20. Deng, D.J., Chang, R.S.: A Priority Scheme for IEEE 802.11 DCF Access Method. IEICE Transactions on Communications E82- B(1), 96-102 (1999)

21. Chang, C.S., Chen, K.C., You, M.Y., Chang, J.F.: Guaranteed Quality-of-Service Wireless Access to ATM Networks. IEEE Journal on Selected Areas of Communications 15(1), 106-118 (1997) 
22. Wang, W., Liew, S.C., Li, V.O.K.: Solutions to Performance Problems in VoIP over a 802.11 Wireless LAN. IEEE Transactions on Vehicular Technology 54(1), 366-384 (2005)

23. IEEE 802.11: The Working Group Setting the Standards for Wireless LANs, http://grouper.ieee.org/groups/802/11/

24. MiTAC International Corporation, "Mio8380" http://www.mitac.com/ 